\title{
A New Numerical Algorithm for Solving a Class of Fractional Advection-Dispersion Equation with Variable Coefficients Using Jacobi Polynomials
}

\author{
A. H. Bhrawy ${ }^{1,2}$ \\ ${ }^{1}$ Department of Mathematics, Faculty of Science, King Abdulaziz University, Jeddah 21589, Saudi Arabia \\ ${ }^{2}$ Department of Mathematics, Faculty of Science, Beni-Suef University, Beni-Suef 62511, Egypt \\ Correspondence should be addressed to A. H. Bhrawy; alibhrawy@yahoo.co.uk
}

Received 17 August 2013; Accepted 17 September 2013

Academic Editor: Dumitru Baleanu

Copyright (C) 2013 A. H. Bhrawy. This is an open access article distributed under the Creative Commons Attribution License, which permits unrestricted use, distribution, and reproduction in any medium, provided the original work is properly cited.

We propose Jacobi-Gauss-Lobatto collocation approximation for the numerical solution of a class of fractional-in-space advectiondispersion equation with variable coefficients based on Caputo derivative. This approach has the advantage of transforming the problem into the solution of a system of ordinary differential equations in time this system is approximated through an implicit iterative method. In addition, some of the known spectral collocation approximations can be derived as special cases from our algorithm if we suitably choose the corresponding special cases of Jacobi parameters $\alpha$ and $\beta$. Finally, numerical results are provided to demonstrate the effectiveness of the proposed spectral algorithms.

\section{Introduction}

Spectral methods have emerged as powerful techniques used in applied mathematics and scientific computing to numerically solve differential equations [1]. Also, they have became increasingly popular for solving fractional differential equations (see, for instance, [2-6]). The main idea of spectral methods is to put the solution of the problem as a sum of certain basis functions and then to choose the coefficients in the sum in order to minimize the difference between the exact solution and approximate one as well as possible. Spectral collocation method has an exponential convergence rate, which is valuable in providing highly accurate solutions to nonlinear differential equations even using a small number of grids. Moreover, the choice of collocation points is very useful for the convergence and efficiency of the collocation approximation $[7,8]$.

In recent years, considerable interest in fractional partial differential equations has been motivated because of their growing applications in electromagnetics, acoustics, viscoelasticity, electrochemistry, and material science [9, 10]. Several analytical algorithms have been investigated for treating these equations analytically to obtain closedform solutions such as variational iteration method, Fourier transform method, homotopy analysis method, the method of separation of variables, Adomian decomposition method, and Laplace transform method [9, 11-14]. However, there are only few types of these equations in which the analytical solutions are available. Therefore, numerical means have to be used in general.

In numerous physical models, an equation commonly used to describe transport diffusive problems is the classical advection-diffusion (or -dispersion) equation which may be generalized to the fractional ones to cover other very interesting physical models. The advection-dispersion equation, which is based on Fick's law, is commonly used to simulate contaminant transport in porous media [15]. The space, time and time-space fractional advection-dispersion equations are presented as a reliable model to simulate the transport of passive tracers carried by fluid flow in a porous media and are used in groundwater hydrology $[13,16]$. Moreover, they have been introduced to describe other important physical phenomena (see [17-21]). 
In the last few years, theory and numerical analysis of fractional partial differential equations have received an increasing attention. In this direction, Rihan [22] proposed the $\theta$-method for approximating time-fractional parabolic partial differential equations in the Caputo sense. An explicit Euler method, an implicit Euler method, and the fractional Cranck-Nicholson method for solving fractional differential equations are discussed in [23-26]. An explicit difference approach for solving space fractional diffusion equation has been proposed in [27]. Ding et al. [28] investigated a class of weighted finite difference method for tackling a class of time-dependent fractional differential equations based on shifted Grünwald formula. K. Wang and H. Wang [29] and Huang et al. [16] proposed a fast numerical scheme for fractional time-dependent advection-diffusion and advectiondispersion equations based on finite difference method, respectively. Recently, the Sinc-Legendre spectral method has been developed in [30] for the fractional convectiondiffusion. Jiang and Lin [31] proposed a new method for a class of fractional advection-dispersion in the reproducing kernel space. Furthermore, Liu et al. [32] proposed an efficient implicit numerical method for a class of fractional advection-dispersion models in which they discussed five fractional models. In the area of numerical methods of fractional partial differential equations, little work has been done by spectral methods compared to finite difference and finite element methods. This partially motivates our interest in such methods.

The main purpose of the this paper is to construct the solution of a class of space fractional advectiondispersion equation with variable coefficients using JacobiGauss-Lobatto collocation (J-GL-C) approximation, based on Jacobi-Gauss-Lobatto quadrature knots, combined with an implicit iterative method for treating the time discretization. More precisely, implementing the J-GL-C approximation to the spatial variable of the fractional advectiondispersion equation and the corresponding boundary conditions reduces the problem to the time integration of a system of ordinary differential equations in respect to the time variable. To the best of our knowledge, such algorithm has not been implemented for solving space fractional initialboundary problems.

The plan of the paper is as follows. In the next section, we introduce basic properties of Jacobi polynomials. In Section 3, the way of constructing the Gauss-Lobatto collocation technique for space fractional advection-dispersion equation with variable coefficients is described using the Jacobi polynomials, and in Section 4 the proposed method is applied to two problems. Finally, some concluding remarks are given in Section 5.

\section{Preliminaries}

In this section, we give some definitions and properties of the fractional calculus (see, e.g., $[9,18,33,34]$ ) and Jacobi polynomials (see, e.g., [35-37]).
For $m$ to be the smallest integer that exceeds $v$, Caputo's fractional derivative operator of order $v>0$ is defined as

$$
\partial_{x}^{v} f(x)= \begin{cases}J^{m-v} D^{m} f(x), & \text { if } m-1<v<m, \\ D^{m} f(x), & \text { if } v=m, m \in N,\end{cases}
$$

where $J^{\nu}$ is the Riemann-Liouville fractional integral operator of order $\nu(\nu \geq 0)$ and is defined as

$$
J^{\nu} f(x)=\frac{1}{\Gamma(\nu)} \int_{0}^{x}(x-t)^{\nu-1} f(t) d t, \quad \nu>0, x>0 .
$$
by

Form (1), the Caputo fractional derivative of $x^{\beta}$ is given

$$
\partial_{x}^{v} x^{\beta}= \begin{cases}0, & \text { for } \beta \in N_{0}, \beta<\lceil\nu\rceil, \\ \frac{\Gamma(\beta+1)}{\Gamma(\beta+1-\nu)} x^{\beta-\nu}, & \text { for } \beta \in N_{0}, \beta \geq\lceil\nu\rceil,\end{cases}
$$

where $\lceil\nu\rceil$ and $\lfloor\nu\rfloor$ are ceiling and floor functions. Also, $N=$ $\{1,2, \ldots\}$ and $N_{0}=\{0,1,2, \ldots\}$. Caputo's fractional differentiation is a linear operation; that is,

$$
\partial_{x}^{\nu}(\lambda f(x)+\mu g(x))=\lambda \partial_{x}^{\nu} f(x)+\mu \partial_{x}^{\nu} g(x),
$$

where $\lambda$ and $\mu$ are constants.

Let $\alpha>-1, \beta>-1$, and $P_{k}^{(\alpha, \beta)}(x)$ be the standard Jacobi polynomial of degree $k$. We have that

$$
\begin{gathered}
P_{k}^{(\alpha, \beta)}(-x)=(-1)^{k} P_{k}^{(\alpha, \beta)}(x), \\
P_{k}^{(\alpha, \beta)}(-1)=\frac{(-1)^{k} \Gamma(k+\beta+1)}{k ! \Gamma(\beta+1)} .
\end{gathered}
$$

For integer $m$, the $m$ th-order derivative of Jacobi polynomials is

$$
D^{m} P_{k}^{(\alpha, \beta)}(x)=2^{-m} \frac{\Gamma(m+k+\alpha+\beta+1)}{\Gamma(k+\alpha+\beta+1)} P_{k-m}^{(\alpha+m, \beta+m)}(x) .
$$

Let $w^{(\alpha, \beta)}(x)=(1-x)^{\alpha}(1+x)^{\beta}$; then we define the weighted space $L_{w^{(\alpha, \beta)}}^{2}(-1,1)$ as usual, equipped with the following inner product and norm:

$$
\begin{gathered}
(u, v)_{w^{(\alpha, \beta)}}=\int_{-1}^{1} u(x) v(x) w^{(\alpha, \beta)}(x) d x, \\
\|v\|_{w^{(\alpha, \beta)}}=(v, v)_{w^{(\alpha, \beta)}}^{1 / 2} .
\end{gathered}
$$

The set of Jacobi polynomials forms a complete $L_{w^{\alpha, \beta}}^{2}(-1,1)$ orthogonal system, and

$$
\begin{aligned}
\left\|P_{k}^{(\alpha, \beta)}\right\|_{w^{(\alpha, \beta)}}^{2} & =h_{k}^{(\alpha, \beta)} \\
& =\frac{2^{\alpha+\beta+1} \Gamma(k+\alpha+1) \Gamma(k+\beta+1)}{(2 k+\alpha+\beta+1) \Gamma(k+1) \Gamma(k+\alpha+\beta+1)} .
\end{aligned}
$$


Let $L>0$; then the shifted Jacobi polynomial of degree $k$ on the interval $(0, L)$ is defined by $P_{L, k}^{(\alpha, \beta)}(x)=P_{k}^{(\alpha, \beta)}((2 x / L)-$ 1).

With the aid of (5), we demonstrate that

$$
P_{L, j}^{(\alpha, \beta)}(0)=(-1)^{j} \frac{\Gamma(j+\beta+1)}{\Gamma(\beta+1) j !} .
$$

Next, let $w_{L}^{(\alpha, \beta)}(x)=(L-x)^{\alpha} x^{\beta}$; then we define the weighted space $L_{w_{L}^{(\alpha, \beta)}}^{2}(0, L)$ in the usual way, with the following inner product and norm:

$$
\begin{gathered}
(u, v)_{w_{L}^{(\alpha, \beta)}}=\int_{0}^{L} u(x) v(x) w_{L}^{(\alpha, \beta)}(x) d x, \\
\|v\|_{w_{L}^{(\alpha, \beta)}}=(v, v)_{w_{L}^{1 / 2}(\alpha, \beta)}^{1 / 2} .
\end{gathered}
$$

The set of shifted Jacobi polynomials is a complete $L_{w_{L}^{(\alpha, \beta)}}^{2}(0, L)$-orthogonal system. Moreover, due to (8), we have

$$
\left\|P_{L, k}^{(\alpha, \beta)}\right\|_{w_{L}^{(\alpha, \beta)}}^{2}=\left(\frac{L}{2}\right)^{\alpha+\beta+1} h_{k}^{(\alpha, \beta)}=h_{L, k}^{(\alpha, \beta)}
$$

\section{Jacobi Spectral Collocation Method}

Since the Jacobi spectral collocation method approximates the initial-boundary problems in physical space and it is a global method, it is very easy to implement and adapt to various problems, including variable coefficient and nonlinear problems (see, for instance, $[7,38]$ ). In this section, a new algorithm for solving time-dependent space fractional advection-dispersion equation is proposed based on JacobiGauss-Lobatto spectral collocation approximation and an implicit iterative method in finite space-time domain.

In this section, we consider the space fractional advection-dispersion equations with space and time variable coefficients $[19,23,28,39,40]$ :

$$
\begin{array}{r}
\frac{\partial u(x, t)}{\partial t}+a(x, t) \frac{\partial u(x, t)}{\partial x}-b(x, t) \frac{\partial^{\nu} u(x, t)}{\partial x^{\nu}}=q(x, t), \\
0 \leq x \leq L, \quad 0<t \leq T, \quad 1<v \leq 2,
\end{array}
$$

subject to the boundary conditions:

$$
\begin{array}{ll}
u(0, t)=g_{0}(x), & 0<t \leq T, \\
u(L, t)=g_{1}(x), & 0<t \leq T,
\end{array}
$$

and the initial value:

$$
u(x, 0)=f(x), \quad 0 \leq x \leq L,
$$

where $a$ is the drift of the process, that is, the mean advective velocity, $b \geq 0$ is the coefficient of dispersion, $v$ is the fractional order in the Caputo sense, $1<v \leq 2$, and $q$ is a source function. In particular, if $v=2$, (12) is the classical convection-diffusion equation with a source term which has commonly been used to describe the Brownian motion of particles [41]. Moreover, if $a=0$, it reduces to the space fractional diffusion equation (cf. [42-46]).

Now we introduce the Jacobi-Gauss-Lobatto quadratures in two different intervals $(-1,1)$, and $(0, L)$. Denote by $x_{N, j}^{(\alpha, \beta)}\left(x_{L, N, j}^{(\alpha, \beta)}\right), 0 \leqslant j \leqslant N$, and $\omega_{N, j}^{(\alpha, \beta)}\left(\omega_{L, N, j}^{(\alpha, \beta)}\right),(0 \leq i \leq N)$, the nodes and Christoffel numbers of the standard (shifted) Jacobi-Gauss-Lobatto quadratures on the intervals $(-1,1)$ and $(0, L)$, respectively. Then one can clearly deduce that

$$
\begin{array}{cl}
x_{L, N, j}^{(\alpha, \beta)}=\frac{L}{2}\left(x_{N, j}^{(\alpha, \beta)}+1\right), & 0 \leq j \leq N, \\
\omega_{L, N, j}^{(\alpha, \beta)}=\left(\frac{L}{2}\right)^{\alpha+\beta+1} \omega_{N, j}^{(\alpha, \beta)}, & 0 \leq j \leq N,
\end{array}
$$

and if $S_{N}(0, L)$ denotes the set of all polynomials of degree at most $N$, then it follows that, for any $\phi \in S_{2 N+1}(0, L)$, we have

$$
\begin{aligned}
& \int_{0}^{L} w_{L}^{(\alpha, \beta)}(x) \phi(x) d x \\
& =\left(\frac{L}{2}\right)^{\alpha+\beta+1} \int_{-1}^{1}(1-x)^{\alpha}(1+x)^{\beta} \\
& \quad \times \phi\left(\frac{L}{2}(x+1)\right) d x \\
& =\left(\frac{L}{2}\right)^{\alpha+\beta+1} \sum_{j=0}^{N} \Phi_{N, j}^{(\alpha, \beta)} \phi\left(\frac{L}{2}\left(x_{N, j}^{(\alpha, \beta)}+1\right)\right) \\
& =\sum_{j=0}^{N} \Phi_{L, N, j}^{(\alpha, \beta)} \phi\left(x_{L, N, j}^{(\alpha, \beta)}\right) .
\end{aligned}
$$

We define the discrete inner product and norm as follows:

$$
\begin{gathered}
(u, v)_{w^{\alpha, \beta}, N}=\sum_{k=0}^{N} u\left(x_{N, k}^{(\alpha, \beta)}\right) v\left(x_{N, k}^{(\alpha, \beta)}\right) \omega_{N, k}^{(\alpha, \beta)}, \\
\|u\|_{w^{\alpha, \beta}, N}=\sqrt{(u, u)_{w^{\alpha, \beta}, N}} .
\end{gathered}
$$

Obviously,

$$
(u, v)_{w^{\alpha, \beta}, N}=(u, v)_{w^{\alpha, \beta}} \quad \forall u v \in S_{2 N-1} .
$$

Thus, for any $u \in S_{N}$, the norms $\|u\|_{w^{\alpha, \beta}, N}$ and $\|u\|_{w^{\alpha, \beta}}$ coincide.

Associating with this quadrature rule, we denote by $I_{N}^{P^{(\alpha, \beta)}}$ the Jacobi-Gauss-Lobatto interpolation (cf. [47]):

$$
I_{N}^{P^{(\alpha, \beta)}} u\left(x_{N, k}^{(\alpha, \beta)}\right)=u\left(x_{N, k}^{(\alpha, \beta)}\right), \quad 0 \leq j \leq N .
$$

We now derive an efficient algorithm for solving spacefractional advection diffusion equation (12)-(14). We expand the numerical approximation in terms of Jacobi polynomials:

$$
u_{N}(x, t)=\sum_{j=0}^{N} a_{j}(t) P_{L, j}^{(\alpha, \beta)}(x) .
$$


If we make use of the orthogonality property of Jacobi polynomials with respect to the weight functions $w^{\alpha, \beta}$ and the discrete inner product (17), then we get

$$
a_{j}(t)=\frac{1}{h_{j}} \sum_{i=0}^{N} P_{L, j}^{(\alpha, \beta)}\left(x_{N, i}^{(\alpha, \beta)}\right) \omega_{N, i}^{(\alpha, \beta)} u\left(x_{N, i}^{(\alpha, \beta)}, t\right),
$$

and accordingly, (20) takes the following form:

$$
\begin{aligned}
u_{N}(x, t)=\sum_{j=0}^{N}\left(\frac{1}{h_{j}} \sum_{i=0}^{N} P_{L, j}^{(\alpha, \beta)}\left(x_{N, i}^{(\alpha, \beta)}\right)\right. & \\
& \left.\times \omega_{N, i}^{(\alpha, \beta)} u\left(x_{N, i}^{(\alpha, \beta)}, t\right)\right) P_{L, j}^{(\alpha, \beta)}(x),
\end{aligned}
$$

or equivalently

$$
\begin{aligned}
u_{N}(x, t)=\sum_{i=0}^{N}\left(\sum_{j=0}^{N}\right. & \frac{1}{h_{j}} P_{L, j}^{(\alpha, \beta)}\left(x_{N, i}^{(\alpha, \beta)}\right) \\
& \left.\times P_{L, j}^{(\alpha, \beta)}(x) \omega_{N, i}^{(\alpha, \beta)}\right) u\left(x_{N, i}^{(\alpha, \beta)}, t\right) .
\end{aligned}
$$

The first order spatial derivative of the spectral solution can be approximated by the J-GL-C points

$$
\begin{aligned}
u_{x}\left(x_{N, n}^{(\alpha, \beta)}, t\right)=\sum_{i=0}^{N}\left(\sum_{j=0}^{N} \frac{1}{h_{j}} P_{j}^{(\alpha, \beta)}\left(x_{N, i}^{(\alpha, \beta)}\right)\right. \\
\left.\quad \times\left(P_{j}^{(\alpha, \beta)}\left(x_{N, n}^{(\alpha, \beta)}\right)\right)^{\prime} \omega_{N, i}^{(\alpha, \beta)}\right) \\
\times u\left(x_{N, i}^{(\alpha, \beta)}, t\right), \quad n=0,1, \ldots, N .
\end{aligned}
$$

According to

$$
D P_{j}^{(\alpha, \beta)}(x)=\frac{\Gamma(j+\alpha+\beta+2)}{2 \Gamma(j+\alpha+\beta+1)} P_{j-1}^{(\alpha+1, \beta+1)}(x),
$$

equation (24) can be written in the following form:

$$
\begin{gathered}
u_{x}\left(x_{N, n}^{(\alpha, \beta)}, t\right) \\
=\sum_{i=0}^{N}\left(\sum_{j=0}^{N} \frac{1}{h_{j}} P_{j}^{(\alpha, \beta)}\left(x_{N, i}^{(\alpha, \beta)}\right) \frac{\Gamma(j+\alpha+\beta+2)}{2 \Gamma(j+\alpha+\beta+1)}\right. \\
\left.\quad \times P_{j-1}^{(\alpha+1, \beta+1)}\left(x_{N, n}^{(\alpha, \beta)}\right) \omega_{N, i}^{(\alpha, \beta)}\right) \\
\times u\left(x_{N, i}^{(\alpha, \beta)}, t\right) \\
=\sum_{i=0}^{N} A_{n i} u\left(x_{N, i}^{(\alpha, \beta)}, t\right), \quad n=0,1, \ldots, N,
\end{gathered}
$$

where

$$
\begin{aligned}
A_{n i}=\sum_{j=0}^{N} & \frac{\Gamma(j+\alpha+\beta+2)}{2 \Gamma(j+\alpha+\beta+1) h_{j}} \\
& \times P_{j}^{(\alpha, \beta)}\left(x_{N, i}^{(\alpha, \beta)}\right) P_{j-1}^{(\alpha+1, \beta+1)}\left(x_{N, n}^{(\alpha, \beta)}\right) \omega_{N, i}^{(\alpha, \beta)} .
\end{aligned}
$$

The fractional derivative of order $v$ in the Caputo sense for the Jacobi polynomials is given by

$$
\begin{array}{r}
\frac{\partial^{\nu}}{\partial x^{\nu}} P_{L, i}^{(\alpha, \beta)}(x)=\sum_{j=0}^{\infty} S_{\nu}(i, j, \alpha, \beta) P_{L, j}^{(\alpha, \beta)}(x), \\
i=\lceil\nu\rceil,\lceil\nu\rceil+1, \ldots,
\end{array}
$$

where

$$
\begin{aligned}
& S_{\nu}(i, j, \alpha, \beta)=\sum_{k=\lceil\nu\rceil}^{i}(-1)^{i-k} L^{\alpha+\beta-\gamma+1} \Gamma(j+\beta+1) \\
& \times \Gamma(i+\beta+1) \Gamma(i+k+\alpha+\beta+1) \\
& \times\left(h_{j} \Gamma(j+\alpha+\beta+1) \Gamma(k+\beta+1)\right. \\
& \times \Gamma(i+\alpha+\beta+1) \\
&\times \Gamma(k-v+1)(i-k) !)^{-1} \\
& \times \sum_{l=0}^{j}(-1)^{j-l} \Gamma(j+l+\alpha+\beta+1) \times \Gamma(\alpha+1) \Gamma(l+k+\beta-v+1) \\
& \times((l+\beta+1) \\
& \times \times \Gamma(l+k+\alpha+\beta-v+2) \\
&\times(j-l) ! l !)^{-1} .
\end{aligned}
$$

The spatial partial fractional derivatives of order $v$ for the spectral solution (20) can be evaluated at the J-GL-C points $\left\{x_{N, n}^{(\alpha, \beta)} ; n=0,1, \ldots, N\right\}$. Hence, we have

$$
\begin{aligned}
& \frac{\partial^{\nu}}{\partial x^{\nu}} u_{N}\left(x_{N, n}^{(\alpha, \beta)}, t\right)=\sum_{i=0}^{N}\left(\sum_{j=0}^{N} \frac{1}{h_{j}} P_{L, j}^{(\alpha, \beta)}\left(x_{N, i}^{(\alpha, \beta)}\right)\right. \\
&\left.\quad \times \frac{\partial^{\nu}}{\partial x^{\nu}}\left(P_{L, j}^{(\alpha, \beta)}\left(x_{N, n}^{(\alpha, \beta)}\right)\right) \omega_{N, i}^{(\alpha, \beta)}\right) \\
& \quad \times u\left(x_{N, i}^{(\alpha, \beta)}, t\right) \\
&=\sum_{i=0}^{N} B_{n i} u\left(x_{N, i}^{(\alpha, \beta)}, t\right), \quad n=0,1, \ldots, N,
\end{aligned}
$$


where

$$
\begin{aligned}
& B_{n i}= \sum_{j=0}^{N} \frac{1}{h_{j}} P_{L, j}^{(\alpha, \beta)}\left(x_{N, i}^{(\alpha, \beta)}\right) \frac{\partial^{\nu}}{\partial x^{\nu}}\left(P_{L, j}^{(\alpha, \beta)}\left(x_{N, n}^{(\alpha, \beta)}\right)\right) \omega_{N, i}^{(\alpha, \beta)} \\
&=\sum_{j=0}^{N} \frac{1}{h_{j}} P_{L, j}^{(\alpha, \beta)}\left(x_{N, i}^{(\alpha, \beta)}\right)\left(\sum_{l=0}^{\infty} S_{\nu}(j, l, \alpha, \beta) P_{L, l}^{(\alpha, \beta)}\left(x_{N, n}^{(\alpha, \beta)}\right)\right) \\
& \quad \times \omega_{N, i}^{(\alpha, \beta)} \\
& \simeq \sum_{j=0}^{N} \sum_{l=0}^{N} \frac{1}{h_{j}} P_{L, j}^{(\alpha, \beta)}\left(x_{N, i}^{(\alpha, \beta)}\right) S_{\nu}(j, l, \alpha, \beta) P_{L, l}^{(\alpha, \beta)}\left(x_{N, n}^{(\alpha, \beta)}\right) \\
& \times \omega_{N, i}^{(\alpha, \beta)},
\end{aligned}
$$

for $1<\nu \leq 2$, and $S_{\nu}(j, l, \alpha, \beta)$ is defined in (29).

If we apply the Jacobi-Gauss-Lobatto collocation method of (12) without the two assigned abscissas 0 and $L ;\left\{x_{N, 0}^{(\alpha, \beta)}=\right.$ $\left.0, x_{N, N}^{(\alpha, \beta)}=L\right\}$, which will be necessary used as two points from the collocation nodes for enforcing the boundary conditions (13), and using (30), then (12) may be written as

$$
\begin{aligned}
u_{t}\left(x_{N, n}^{(\alpha, \beta)}, t\right)= & -a\left(x_{N, n}^{(\alpha, \beta)}, t\right) \sum_{i=0}^{N} A_{n i} u\left(x_{N, i}^{(\alpha, \beta)}, t\right) \\
& -b\left(x_{N, n}^{(\alpha, \beta)}, t\right) \sum_{i=0}^{N} B_{n i} u\left(x_{N, i}^{(\alpha, \beta)}, t\right) \\
& +q\left(x_{N, n}^{(\alpha, \beta)}, t\right), \quad n=1, \ldots, N-1 .
\end{aligned}
$$

Let us denote that

$$
\begin{aligned}
& u_{n}(t)=u_{N}\left(x_{N, n}^{(\alpha, \beta)}, t\right), \\
& a_{n}(t)=a\left(x_{N, n}^{(\alpha, \beta)}, t\right), \\
& b_{n}(t)=b\left(x_{N, n}^{(\alpha, \beta)}, t\right), \\
& q_{n}(t)=q\left(x_{N, n}^{(\alpha, \beta)}, t\right), \\
& \dot{u}_{n}(t)=u_{t}\left(x_{N, n}^{(\alpha, \beta)}, t\right) .
\end{aligned}
$$

Thus, (32) can be rewritten in the following simple form:

$$
\begin{array}{r}
\dot{u}_{n}(t)=-a_{n}(t) \sum_{i=0}^{N} A_{n i} u_{i}(t) \\
-b_{n}(t) \sum_{i=0}^{N} B_{n i} u_{i}(t)+q_{n}(t), \\
n=1, \ldots, N-1 .
\end{array}
$$

Let us assume that

$$
\begin{aligned}
& d_{n}(t)=a_{n}(t)\left(A_{n 0} g_{1}(t)+A_{n N} g_{2}(t)\right), \\
& e_{n}(t)=b_{n}(t)\left(B_{n 0} g_{1}(t)+B_{n N} g_{2}(t)\right),
\end{aligned}
$$

Then (34) and using the two-point boundary conditions (13) generate a system of $(N-1)$ ordinary differential equations in time.

$$
\begin{aligned}
\dot{u}_{n}(t)= & -a_{n}(t) \sum_{i=1}^{N-1} A_{n i} u_{i}(t) \\
& -b_{n}(t) \sum_{i=1}^{N-1} B_{n i} u_{i}(t) \\
& -d_{n}(t)-e_{n}(t)+q_{n}(t), \quad n=1, \ldots, N-1 .
\end{aligned}
$$

with the initial values

$$
u_{n}(0)=f\left(x_{N, n}^{(\alpha, \beta)}\right), \quad n=1, \ldots, N-1,
$$

which may be written in the following matrix form:

$$
\begin{gathered}
\dot{\mathbf{u}}(t)=\mathbf{F}(t, u(t)), \\
\mathbf{u}(0)=\mathbf{f},
\end{gathered}
$$

where

$$
\begin{gathered}
\dot{\mathbf{u}}(t)=\left[\dot{u}_{1}(t), \dot{u}_{2}(t), \ldots, \dot{u}_{N-1}(t)\right]^{T}, \\
\mathbf{f}=\left[f\left(x_{N, 1}\right), f\left(x_{N, 2}\right), \ldots, f\left(x_{N, N-1}\right)\right], \\
\mathbf{F}(t, u(t))=\left[F_{1}(t, u(t)), F_{2}(t, u(t)), \ldots, F_{N-1}(t, u(t))\right]^{T}, \\
F_{n}(t, u(t))=-a_{n}(t) \sum_{i=1}^{N-1} A_{n i} u_{i}(t) \\
-b_{n}(t) \sum_{i=1}^{N-1} B_{n i} u_{i}(t) \\
\quad-d_{n}(t)-e_{n}(t)+q_{n}(t), \quad n=1, \ldots, N-1 .
\end{gathered}
$$

The system of ordinary differential equations (38) in time may be solved using any standard technique to find $u_{n}(t)$ and then $u_{N}(x, t)$ from (22).

\section{Numerical Results}

In order to check the accuracy and reliability of the proposed algorithm, we present two numerical examples using the proposed algorithm. In the first example, we compute the space fractional diffusion equation to check the accuracy, and space fractional advection-dispersion equation with variable coefficients is solved in the second example which confirms the good accuracy of our method. Comparing the results obtained by various choices of Jacobi parameters $\alpha$ and $\beta$ and results presented elsewhere reveals that the present method is very effective and convenient for all choices of $\alpha$ and $\beta$.

Example 1. Consider the space fractional diffusion equation (see, $[42,48,49])$ :

$$
\begin{array}{r}
\frac{\partial u(x, t)}{\partial t}-\frac{\Gamma(2.2) x^{2.8}}{6} \frac{\partial^{1.8} u(x, t)}{\partial x^{1.8}}=-(1+x) e^{-t} x^{3}, \\
x \in(0,1), t \in(0, T],
\end{array}
$$


TABLE 1: Comparing maximum absolute errors of the proposed method and [42, 49].

\begin{tabular}{|c|c|c|c|c|c|c|}
\hline$N$ & $\alpha=\beta=1 / 2$ & $\alpha=-\beta=1 / 2$ & $-\alpha=\beta=1 / 2$ & $\mathrm{CN}$ [42] & Extra CN [42] & BEFD [49] \\
\hline 3 & $1.04 \times 10^{-1}$ & $1.45 \times 10^{-1}$ & $1.45 \times 10^{-1}$ & - & - & - \\
\hline 5 & $8.51 \times 10^{-6}$ & $4.28 \times 10^{-5}$ & $7.33 \times 10^{-6}$ & - & - & - \\
\hline 10 & $3.75 \times 10^{-7}$ & $2.85 \times 10^{-6}$ & $3.60 \times 10^{-7}$ & $1.82 \times 10^{-3}$ & $1.77 \times 10^{-4}$ & $8.05 \times 10^{-3}$ \\
\hline 15 & $5.86 \times 10^{-8}$ & $5.77 \times 10^{-7}$ & $8.59 \times 10^{-8}$ & $1.16 \times 10^{-3}$ & $7.85 \times 10^{-5}$ & $5.48 \times 10^{-3}$ \\
\hline 20 & $3.03 \times 10^{-8}$ & $1.79 \times 10^{-7}$ & $3.47 \times 10^{-8}$ & $8.64 \times 10^{-4}$ & $4.40 \times 10^{-5}$ & $4.24 \times 10^{-3}$ \\
\hline 25 & $1.97 \times 10^{-8}$ & $7.11 \times 10^{-8}$ & $3.20 \times 10^{-8}$ & - & - & - \\
\hline
\end{tabular}

TABLE 2: Comparing maximum absolute errors for different choices of $a l$ and $\beta$ and $N=3,6,12,24$.

\begin{tabular}{lccccc}
\hline$N$ & $\alpha=\beta=1 / 2$ & $\alpha=-\beta=1 / 2$ & $-\alpha=\beta=1 / 2$ & $\alpha=\beta=0$ & $\alpha=\beta=3 / 2$ \\
\hline 3 & $1.99 \times 10^{-2}$ & $3.40 \times 10^{-2}$ & $2.30 \times 10^{-2}$ & $1.30 \times 10^{-2}$ & $3.48 \times 10^{-4}$ \\
6 & $6.10 \times 10^{-4}$ & $1.45 \times 10^{-3}$ & $6.84 \times 10^{-4}$ & $1.04 \times 10^{-4}$ & $1.21 \times 10^{-3}$ \\
12 & $1.02 \times 10^{-4}$ & $5.08 \times 10^{-4}$ & $1.05 \times 10^{-4}$ & $5.41 \times 10^{-5}$ & $1.06 \times 10^{-4}$ \\
18 & $5.38 \times 10^{-5}$ & $2.61 \times 10^{-4}$ & $5.47 \times 10^{-5}$ & $3.92 \times 10^{-5}$ & $3.74 \times 10^{-5}$ \\
24 & $3.55 \times 10^{-5}$ & $1.34 \times 10^{-4}$ & $3.49 \times 10^{-5}$ & \\
\hline
\end{tabular}

with the initial condition:

$$
u(x, 0)=x^{3} \quad \text { for } x \in(0,1)
$$

and the boundary conditions:

$$
u(0, t)=0, \quad u(1, t)=e^{-t}, \quad t \in(0, T] .
$$

The exact solution to this problem is

$$
u(x, t)=e^{-t} x^{3}
$$

In Table 1, we list the maximum absolute errors using JGL-C method with three choices of the Jacobi parameters $\alpha$ and $\beta$ and various choices of the $N$.

We contrast our results with the corresponding results for the backward Euler finite difference scheme (BEFD [49]), the fractional Crank-Nicholson approach (CN [50]), and the extrapolated fractional Crank-Nicholson approach (Extra CN [50]) which we have presented in the fifth, sixth, and seventh columns of Table 1 . We should note that for all values of $\alpha$ and $\beta$, the proposed method is always more accurate than the results of CN [50], Extra CN [50], and BEFD [49], which shows the spectral accuracy of our method.

In Figures 1 and 2, the analytical solutions and the numerical solutions for $x \in(0,1), t=0.1,0.5,0.7,0.9$, and $x=0.2,0.6,0.8,1.0, t \in(0,1)$, are shown, respectively. Consequently, we see that all numerical solutions are in complete agreement with the analytical solutions.

Example 2. Consider the space fractional advection-dispersion equation with variable coefficients:

$$
\begin{array}{r}
\frac{\partial u(x, t)}{\partial t}=-\frac{t x}{\theta} \frac{\partial u(x, t)}{\partial x}+\frac{t^{2} x^{\theta}}{\Gamma(1+\theta)} \frac{\partial^{\theta} u(x, t)}{\partial x^{\theta}}+f(x, t) \\
x \in[0,2], \quad t \in(0, T]
\end{array}
$$

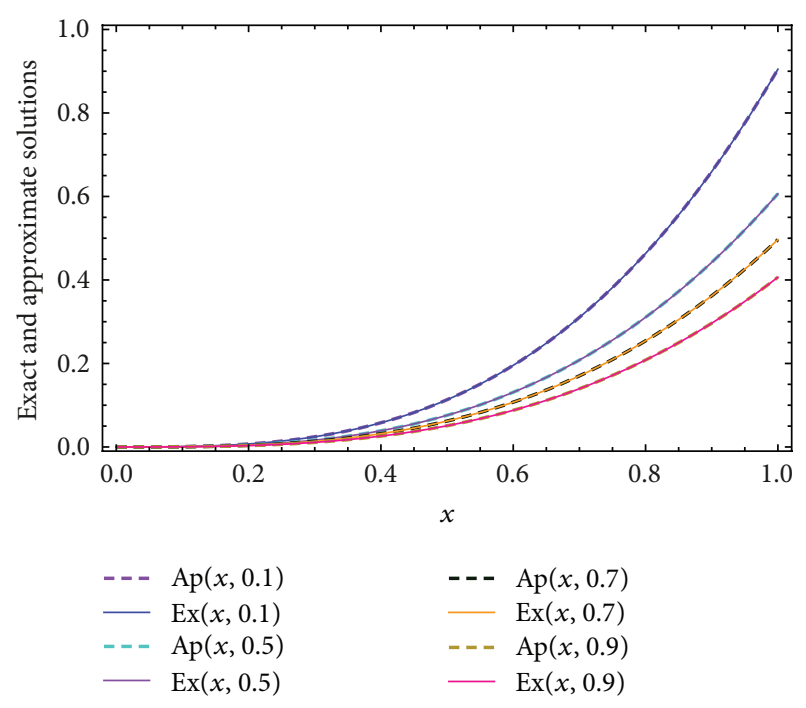

FIGURE 1: The comparison of the curves of analytical solutions and approximate solutions at $N=24$ and $t=0.1,0.5,0.7,0.9$.

where

$$
f(x, t)=x^{\theta}\left(4 \pi \cos (4 \pi t)+t \sin (4 \pi t)-t^{2} \sin (4 \pi t)\right),
$$

with the initial condition:

$$
u(x, 0)=0 \quad \text { for } x \in[0,2]
$$

and the boundary conditions:

$$
u(0, t)=0, \quad u(2, t)=2^{\theta} \sin (4 \pi t), \quad t \in(0, T] .
$$

The exact solution to this problem is

$$
u(x, t)=\sin (4 \pi t) x^{\theta}
$$




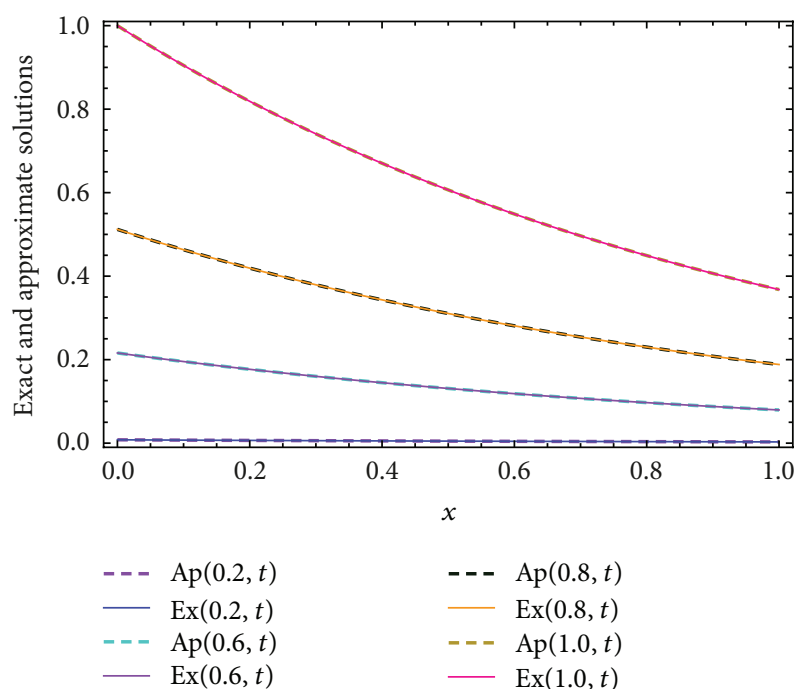

FIgURE 2: The comparison of the curves of analytical solutions and approximate solutions at $N=24$ and $x=0.2,0.6,0.8,1.0$.

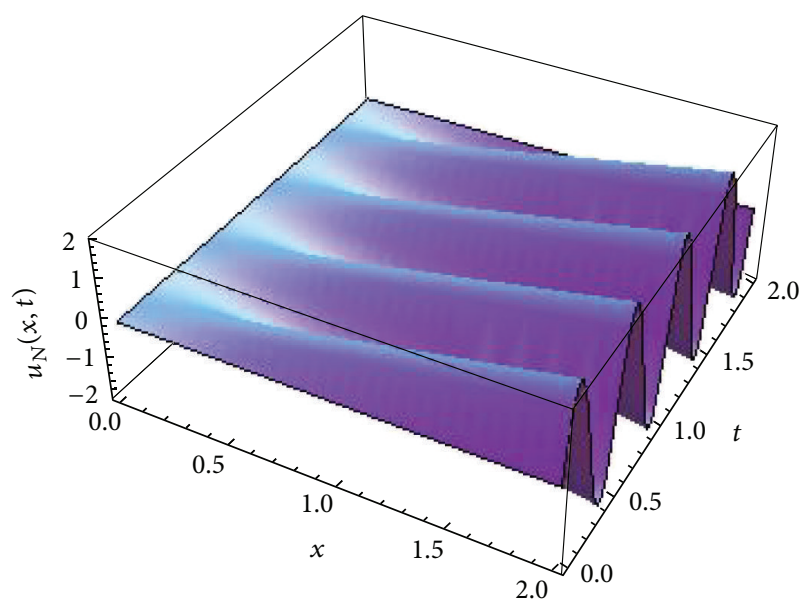

FIGURE 3: The space-time graph of approximate solutions at $\theta=$ 1.01 .

For the sake of comparison of some different values of Jacobi parameters $\alpha$ and $\beta$, we introduce in Table 2 the maximum absolute errors between the exact and numerical solutions using Jacobi Gauss-Lobatto collocation method with $\theta=1.9, t \in(0,2]$, and various choices of $N$. Consequently, we conclude that all numerical solutions are in good agreement with the analytical solutions in all choices of $\alpha$ and $\beta$.

In case of Chebyshev polynomials of the first kind $\alpha=$ $\beta=-1 / 2$, the space-time graphs of approximate solutions at $N=28$ for the two choices $\theta=1.01$ and $\theta=1.81$ are shown in Figures 3 and 4, respectively. From these figures, it can be seen that the numerical solutions are in excellent agreement with the exact solutions. Numerical simulation is given in Figure 5 to compare the curves of exact solution and approximate solution (in case of Legendre polynomials $\alpha=\beta=0$ and $N=16)$ for $\theta=1.5$ with $x \in(0,2)$ and

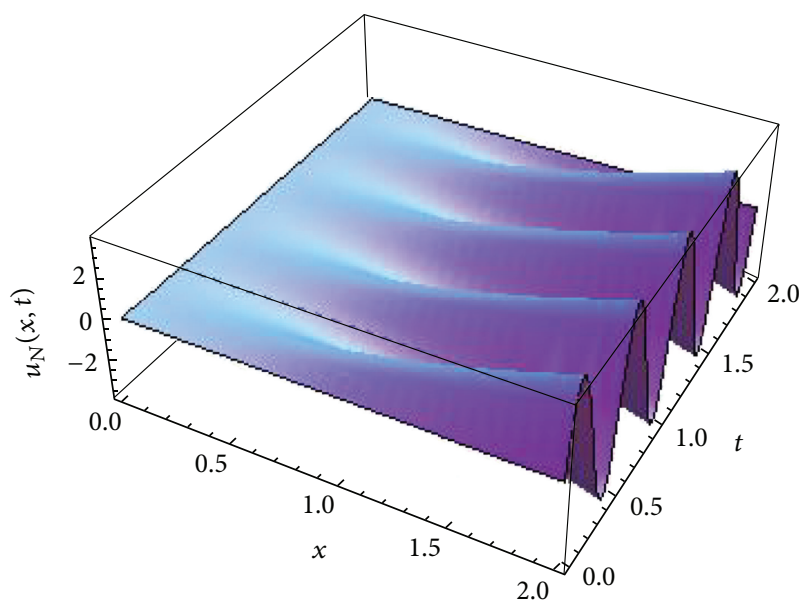

FIGURE 4: The space-time graph of approximate solutions at $\theta=$ 1.81 .

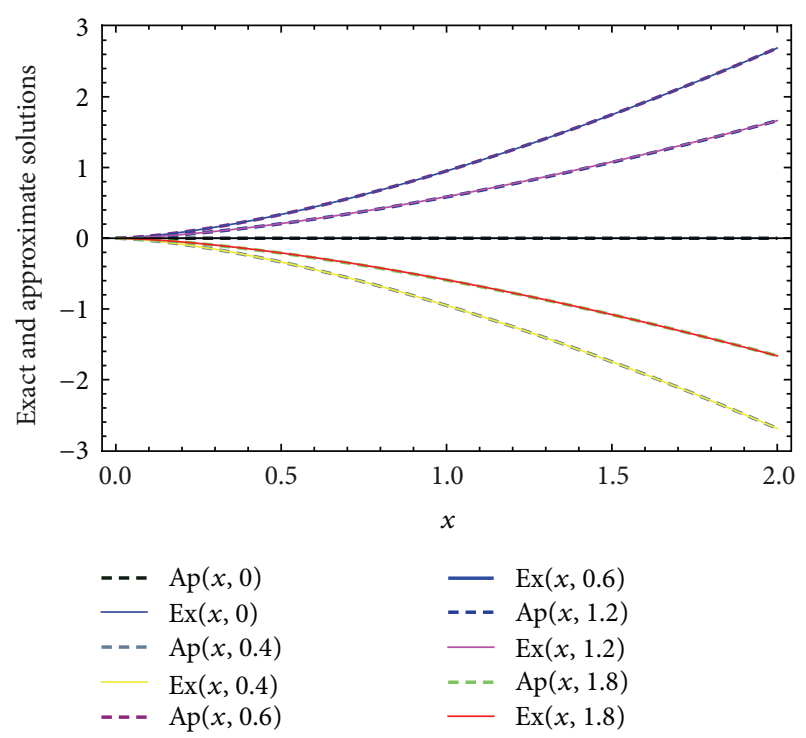

FIgURE 5: The comparison of the curves of analytical solutions and approximate solutions at $t=0,0.4,0.6,1.2,1.8$.

$t=0,0.4,0.6,1.2,1.8$. Moreover, the curves of exact solution and approximate solution (in case of Chebyshev polynomials of the second kind $\alpha=\beta=1 / 2$ and $N=16$ ) for $\theta=1.5$ with $t \in(0,2)$ and $x=0.2,0.6,1.2,1.6,2$ are sketched in Figure 6. Consequently, we see that the curves of the exact and approximate solutions almost coincide for all chosen values of $t$ and $x$.

The obtained results of this example show that the Jacobi Gauss-Lobatto collocation method is simple and very accurate for all values of $\alpha$ and $\beta$. Also by selecting limited Gauss-Lobatto collocation points, excellent numerical results are obtained. 


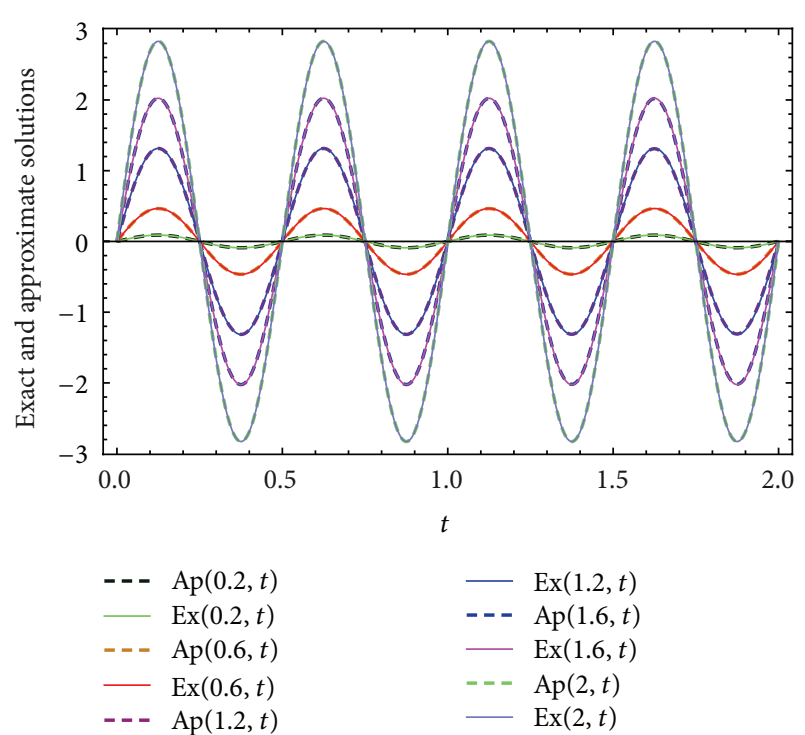

FIgURE 6: The comparison of the curves of analytical solutions and approximate solutions at $x=0.2,0.6,1.2,1.6,2$.

\section{Conclusion and Future Work}

In this paper, we have proposed the Jacobi Gauss-Lobatto collocation spectral approximation for tackling fractionalin-space advection-dispersion equation subject to initialboundary conditions. Applying the collocation method has reduced the problem to system of ordinary differential equations in time. This system may be solved by an implicit iterative technique. One of the main advantages of the proposed method is the Legendre Gauss-Lobatto collocation approximation, and the four kinds of Chebyshev GaussLobatto collocation approximations may be obtained as special cases of the proposed Jacobi Gauss-Lobatto collocation approximation by taking the corresponding special cases of the Jacobi parameters $\alpha$ and $\beta$. The numerical results given in Section 4 demonstrate the good accuracy of proposed algorithm.

The implementation of Jacobi Gauss-Lobatto collocation spectral approximation for time-space fractional advectiondispersion equations may also constitute another line of our future lines of research. We also conclude that this algorithm can be useful in dealing with coupled nonlinear partial differential equations.

\section{Conflict of Interests}

The author declares that there is no conflict of interests regarding the publication of this paper.

\section{Acknowledgments}

This work was funded by the Deanship of Scientific Research (DSR), King Abdulaziz University, Jeddah, Saudi Arabia under Grant no. (130-141-D1433). The author, therefore, acknowledges the DSR technical and financial support.

\section{References}

[1] C. Canuto, M. Y. Hussaini, A. Quarteroni, and T. A. Zang, Spectral Methods: Fundamentals in Single Domains, Springer, New York, NY, USA, 2006.

[2] D. Baleanu, A. H. Bhrawy, and T. M. Taha, "Two efficient generalized Laguerre spectral algorithms for fractional initial value problems," Abstract and Applied Analysis, vol. 2013, Article ID 546502, 10 pages, 2013.

[3] D. Baleanu, A. H. Bhrawy, and T. M. Taha, "A modified generalized Laguerre spectral methods for fractional differential equations on the half line," Abstract and Applied Analysis, vol. 2013, Article ID 413529, 12 pages, 2013.

[4] A. H. Bhrawy and A. S. Alofi, "The operational matrix of fractional integration for shifted Chebyshev polynomials," Applied Mathematics Letters, vol. 26, no. 1, pp. 25-31, 2013.

[5] E. H. Doha, A. H. Bhrawy, and S. S. Ezz-Eldien, "A new Jacobi operational matrix: an application for solving fractional differential equations," Applied Mathematical Modelling, vol. 36, no. 10, pp. 4931-4943, 2012.

[6] J. A. Rad, S. Kazem, M. Shaban, K. Parand, and A. Yildirim, "Numerical solution of fractional differential equations with a Tau method based on Legendre and Bernstein polynomials," Mathematical Methods in the Applied Sciences, 2013.

[7] A. H. Bhrawy and A. S. Alofi, "A Jacobi-Gauss collocation method for solving nonlinear Lane-Emden type equations," Communications in Nonlinear Science and Numerical Simulation, vol. 17, no. 1, pp. 62-70, 2012.

[8] R. Mohammadi, "B-spline collocation algorithm for numerical solution of the generalized Burger's-Huxley equation," Numerical Methods for Partial Differential Equations, vol. 29, no. 4, pp. 1173-1191, 2013.

[9] I. Podlubny, Fractional Differential Equations, Academic Press Inc., New York, NY, USA, 1999.

[10] Y. Zhang, D. A. Benson, and D. M. Reeves, “Time and space nonlocalities underlying fractional-derivative models: distinction and literature review of field applications," Advances in Water Resources, vol. 32, no. 4, pp. 561-581, 2009.

[11] R. K. Pandey, Om. P. Singh, and V. K. Baranwal, "An analytic algorithm for the space-time fractional advection-dispersion equation," Computer Physics Communications, vol. 182, no. 5, pp. 1134-1144, 2011.

[12] Rajeev and M. S. Kushwaha, "Homotopy perturbation method for a limit case Stefan problem governed by fractional diffusion equation," Applied Mathematical Modelling, vol. 37, no. 5, pp. 3589-3599, 2013.

[13] A. Golbabai and K. Sayevand, "Analytical modelling of fractional advection-dispersion equation defined in a bounded space domain," Mathematical and Computer Modelling, vol. 53, no. 9-10, pp. 1708-1718, 2011.

[14] S. Momani and Z. Odibat, "Numerical solutions of the space-time fractional advection-dispersion equation," Numerical Methods for Partial Differential Equations, vol. 24, no. 6, pp. 1416-1429, 2008.

[15] J. Bear, Hydraulics of Groundwater, McGraw-Hill, New York, NY, USA, 1979.

[16] Q. Huang, G. Huang, and H. Zhan, "A finite element solution for the fractional advection-dispersion equation," Advances in Water Resources, vol. 31, no. 12, pp. 1578-1589, 2008.

[17] R. Hilfer, Ed., Applications of Fractional Calculus in Physics, World Scientific, Singapore, 2000. 
[18] F. Mainardi, "Fractional calculus: some basic problems in continuum and statistical mechanics," in Fractals and Fractional Calculus in Continuum Mechanics, A. Carpinteri and F. Mainardi, Eds., vol. 378, pp. 291-348, Springer, New York, NY, USA, 1997.

[19] R. Metzler and J. Klafter, "The random walk's guide to anomalous diffusion: a fractional dynamics approach," Physics Reports, vol. 339, no. 1, pp. 1-77, 2000.

[20] R. Metzler and J. Klafter, "The restaurant at the end of the random walk: recent developments in the description of anomalous transport by fractional dynamics," Journal of Physics A, vol. 37, no. 31, pp. R161-R208, 2004.

[21] V. V. Uchaikin, Method of Fractional Derivatives, Artishok, Uljanovsk, Russia, 2008.

[22] F. A. Rihan, "Computational methods for delay parabolic and time-fractional partial differential equations," Numerical Methods for Partial Differential Equations, vol. 26, no. 6, pp. 1556-1571, 2010.

[23] M. M. Meerschaert and C. Tadjeran, "Finite difference approximations for fractional advection-dispersion flow equations," Journal of Computational and Applied Mathematics, vol. 172, no. 1, pp. 65-77, 2004.

[24] M. M. Meerschaert and C. Tadjeran, "Finite difference approximations for two-sided space-fractional partial differential equations," Applied Numerical Mathematics, vol. 56, no. 1, pp. 80-90, 2006.

[25] M. M. Meerschaert, H.-P. Scheffler, and C. Tadjeran, "Finite difference methods for two-dimensional fractional dispersion equation," Journal of Computational Physics, vol. 211, no. 1, pp. 249-261, 2006.

[26] C. Tadjeran and M. M. Meerschaert, "A second-order accurate numerical method for the two-dimensional fractional diffusion equation," Journal of Computational Physics, vol. 220, no. 2, pp. 813-823, 2007.

[27] S. Shen and F. Liu, "Error analysis of an explicit finite difference approximation for the space fractional diffusion equation with insulated ends," The ANZIAM Journal, vol. 46, pp. C871-C887, 2005.

[28] Z. Ding, A. Xiao, and M. Li, "Weighted finite difference methods for a class of space fractional partial differential equations with variable coefficients," Journal of Computational and Applied Mathematics, vol. 233, no. 8, pp. 1905-1914, 2010.

[29] K. Wang and H. Wang, "A fast characteristic finite difference method for fractional advection-diffusion equations," Advances in Water Resources, vol. 34, no. 7, pp. 810-816, 2011.

[30] A. Saadatmandi, M. Dehghan, and M.-R. Azizi, "The sincLegendre collocation method for a class of fractional convection-diffusion equations with variable coefficients," Communications in Nonlinear Science and Numerical Simulation, vol. 17, no. 11, pp. 4125-4136, 2012.

[31] W. Jiang and Y. Lin, "Approximate solution of the fractional advection-dispersion equation," Computer Physics Communications, vol. 181, no. 3, pp. 557-561, 2010.

[32] F. Liu, P. Zhuang, and K. Burrage, "Numerical methods and analysis for a class of fractional advection-dispersion models," Computers \& Mathematics with Applications, vol. 64, no. 10, pp. 2990-3007, 2012.

[33] S. Abdi-mazraeh, M. Lakestani, and M. Dehghan, "The construction of operational matrices of integral and fractional integral using the flatlet oblique multiwavelets," Journal of Vibration and Control, 2013.
[34] D. Baleanu, K. Diethelm, E. Scalas, and J. J. Trujillo, Fractional Calculus Models and Numerical Methods, vol. 3 of Complexity, Nonlinearity and Chaos, World Scientific Publishing, Singapore, 2012.

[35] Szegő and Gábor, Orthogonal Polynomials, Colloquium Publications. XXIII. American Mathematical Society, 1939.

[36] E. H. Doha and A. H. Bhrawy, "Efficient spectral-Galerkin algorithms for direct solution of fourth-order differential equations using Jacobi polynomials," Applied Numerical Mathematics, vol. 58 , no. 8, pp. 1224-1244, 2008.

[37] E. H. Doha, A. H. Bhrawy, and R. M. Hafez, "A JacobiJacobi dual-Petrov-Galerkin method for third- and fifth-order differential equations," Mathematical and Computer Modelling, vol. 53, no. 9-10, pp. 1820-1832, 2011.

[38] A. H. Bhrawy, "A. Jacobi-Gauss-Lobatto Collocation method for solving generalized Fitzhugh-Nagumo equation with timedependent coefficients," Applied Mathematics and Computation, vol. 222, pp. 255-264, 2013.

[39] L. Su, W. Wang, and Q. Xu, "Finite difference methods for fractional dispersion equations," Applied Mathematics and Computation, vol. 216, no. 11, pp. 3329-3334, 2010.

[40] G. J. Fix and J. P. Roop, "Least squares finite-element solution of a fractional order two-point boundary value problem," Computers \& Mathematics with Applications, vol. 48, no. 7-8, pp. 1017-1033, 2004.

[41] H. Risken, The Fokker Planck Equation, Springer, Berlin, Germany, 1988.

[42] C. Tadjeran, M. M. Meerschaert, and H.-P. Scheffler, "A secondorder accurate numerical approximation for the fractional diffusion equation," Journal of Computational Physics, vol. 213, no. 1, pp. 205-213, 2006.

[43] E. Sousa, "Numerical approximations for fractional diffusion equations via splines," Computers \& Mathematics with Applications, vol. 62, no. 3, pp. 938-944, 2011.

[44] A. Saadatmandi and M. Dehghan, "A tau approach for solution of the space fractional diffusion equation," Computers \& Mathematics with Applications, vol. 62, no. 3, pp. 1135-1142, 2011.

[45] E. A. Abdel-Rehim and R. Gorenflo, "Simulation of the continuous time random walk of the space-fractional diffusion equations," Journal of Computational and Applied Mathematics, vol. 222, no. 2, pp. 274-283, 2008.

[46] E. H. Doha, A. H. Bhrawy, and S. S. Ezz-Eldien, "Efficient Chebyshev spectral methods for solving multi-term fractional orders differential equations," Applied Mathematical Modelling, vol. 35, no. 12, pp. 5662-5672, 2011.

[47] B.-Y. Guo and L.-I. Wang, "Jacobi approximations in nonuniformly Jacobi-weighted Sobolev spaces," Journal of Approximation Theory, vol. 128, no. 1, pp. 1-41, 2004.

[48] S. S. Ray, K. S. Chaudhuri, and R. K. Bera, "Application of modified decomposition method for the analytical solution of space fractional diffusion equation," Applied Mathematics and Computation, vol. 196, no. 1, pp. 294-302, 2008.

[49] H. W. Choi, S. K. Chung, and Y. J. Lee, "Numerical solutions for space fractional dispersion equations with nonlinear source terms," Bulletin of the Korean Mathematical Society, vol. 47, no. 6, pp. 1225-1234, 2010.

[50] E. Sousa, "A second order explicit finite difference method for the fractional advection diffusion equation," Computers \& Mathematics with Applications, vol. 64, no. 10, pp. 3141-3152, 2012. 


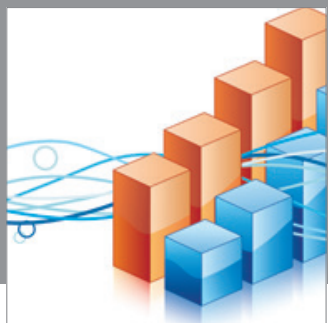

Advances in

Operations Research

mansans

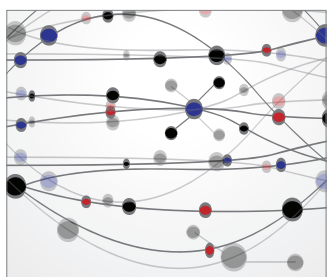

The Scientific World Journal
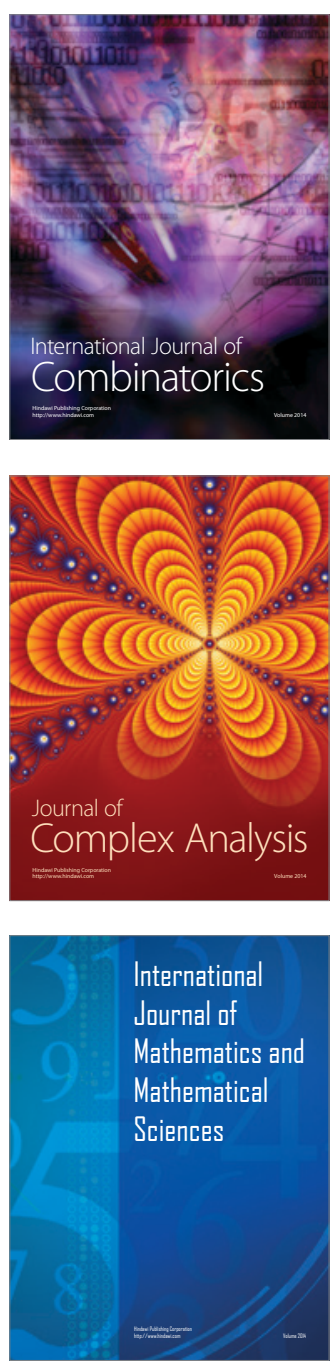
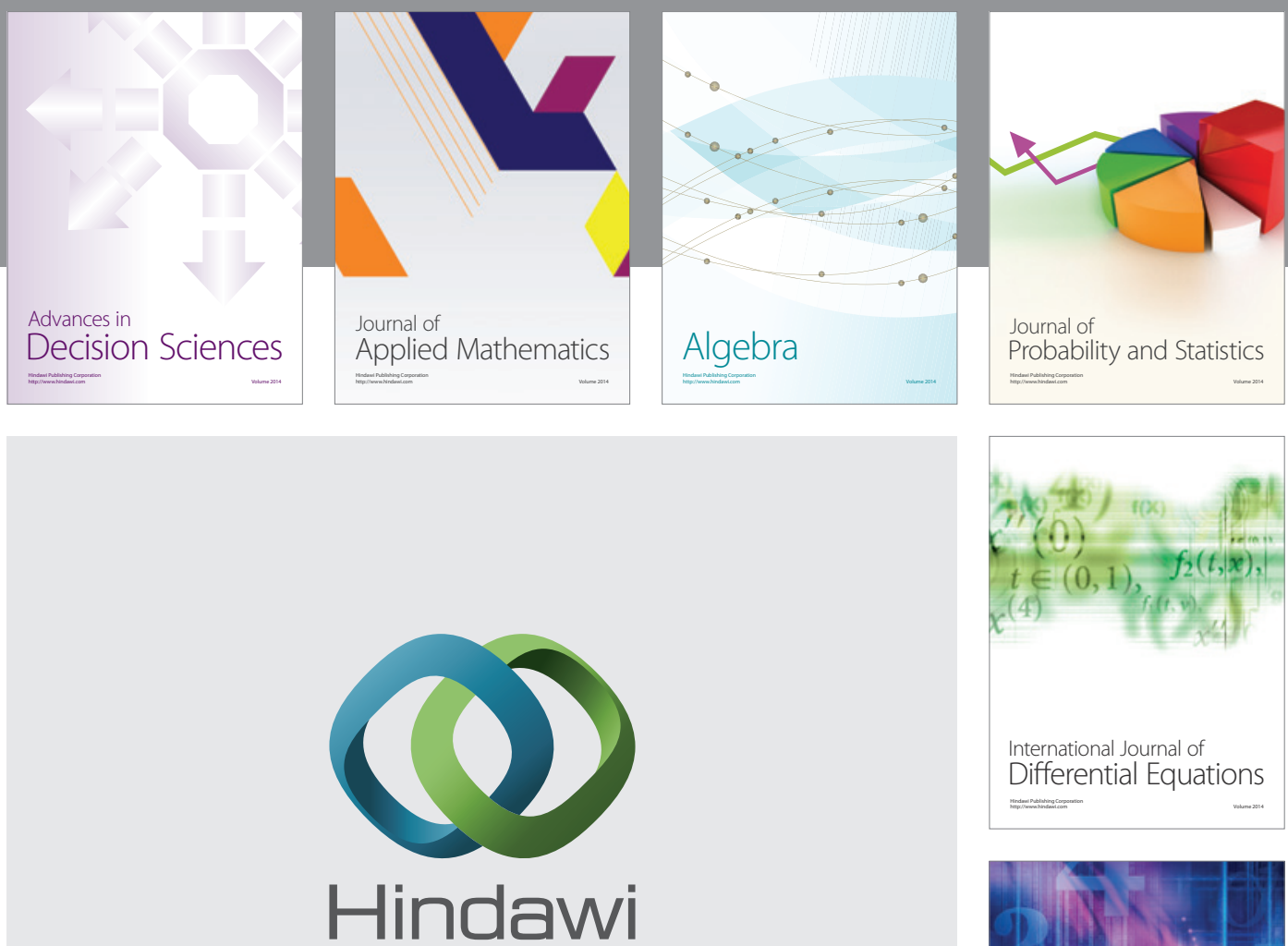

Submit your manuscripts at http://www.hindawi.com
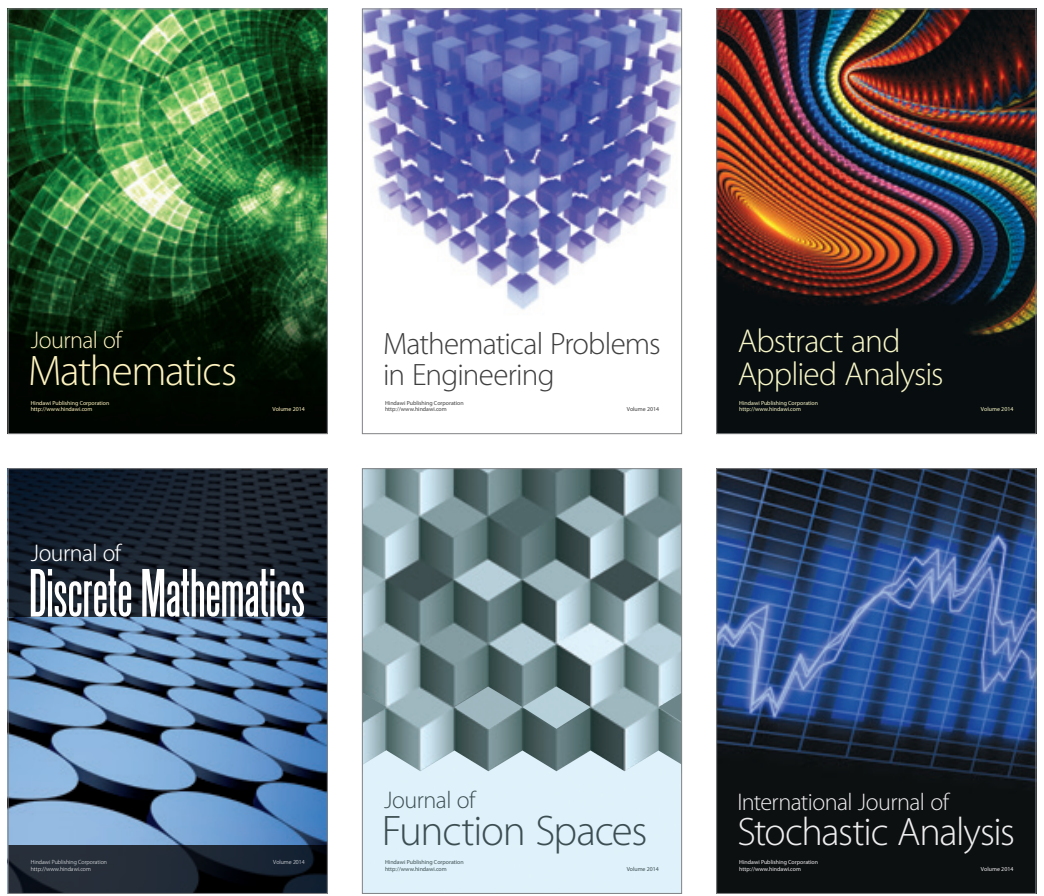

Journal of

Function Spaces

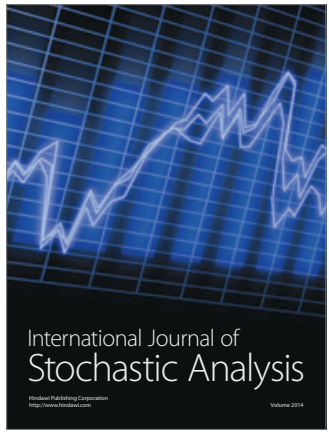

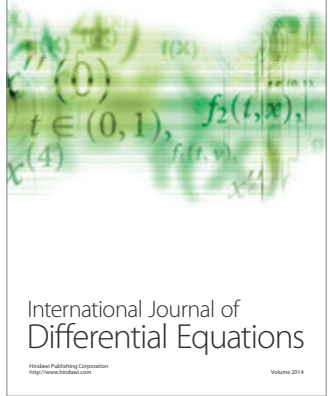
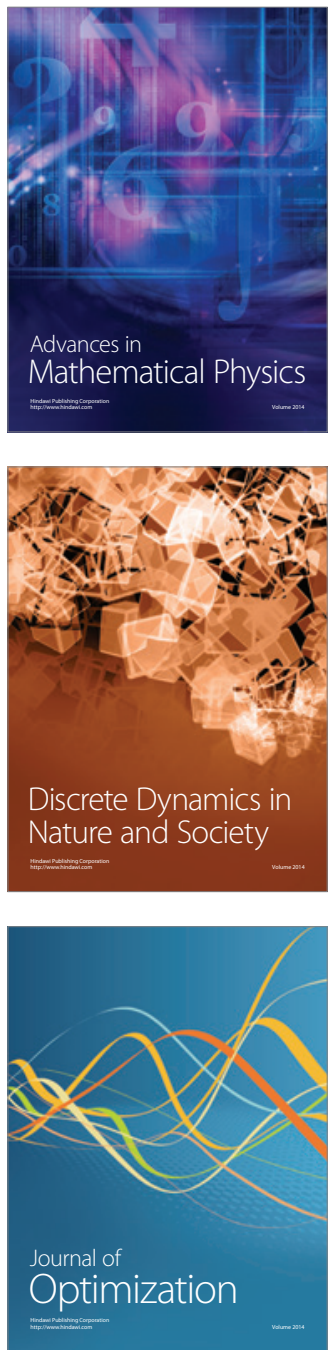